\title{
Quorums-based Replication of Multimedia Objects in Distributed Systems
}

\author{
Tadateru Ohkawara $^{1 *}$, Ailixier Aikebaier ${ }^{3}$, Tomoya Enokido $^{2}$ and Makoto Takizawa ${ }^{1}$
}

\author{
*Correspondence: \\ tadateru.ohkawara@gmail.com \\ 1 Department of Computer and \\ Information Science, Seikei \\ University, 3-3-1 Kichijoji-kitamachi, \\ Musashino-shi, Tokyo 180-8633, \\ Japan \\ Full list of author information is \\ available at the end of the article
}

\begin{abstract}
Background: Multimedia objects like music and movies are distributed to peers through downloading and caching in peer-to-peer (P2P) overlay networks. In this paper, we consider multimedia objects which are characterized in terms of not only data structure but also quality of service (QoS) like frame rate and number of colours. For example, there are a pair of replicas $o_{i}$ and $o_{j}$ of a fully coloured movie object $o$. Here, a content of a replica $o_{i}$ is changed by adding a subobject but another replica $o_{j}$ is not changed. On the other hand, the colour of the replica $o_{j}$ is changed with monochromatic one but not in the replica $o_{j}$. This means, the replica $o_{j}$ is newer than the replica $o_{j}$ with respect to the content but is older than $o_{j}$ with respect to QoS. Thus, replicas of a multimedia object are partially ordered in terms of newness of not only content but also QoS parameters.
\end{abstract}

Methods: In traditional quorum-based (QB) protocols, replicas are totally ordered just in terms of newness of content. We discuss a multimedia quorum-based (MQB) protocol to synchronize multiple replicas to make consistent on the basis of the newness-precedent relation of replicas. Here, the replicas are ordered in vectors of version counters of content and QoS parameters. Every replica in a quorum is not updated for QoS operations to reduce the communication overhead. We evaluate the MQB protocol in terms of communication overhead and show the communication overhead can be reduced in the MQB protocol compared with the traditional QB protocol.

Conclusions: We discussed the multimedia quorum-based (MQB) protocol to keep replicas of a multimedia object mutually consistent. We evaluated the MQB protocol in terms of the total volume of data transmitted among the replicas. Then, we showed the total amount of data transmitted can be reduced in the MQB protocol compared with the traditional quorum-based (QB) protocol.

\section{望 Springer}

\section{Background}

In scalable distributed systems like cloud computing systems [1] and peer-to-peer (P2P) overlay networks [2] systems, resource objects like databases and files are replicated and distributed to multiple server computers in order to increase the performance, reliability, and availability. In P2P overlay networks, objects, especially multimedia objects like movies are in nature autonomously distributed through peer-to-peer communication. There are many discussions on how to maintain the mutual consistency of multiple replicas like the two-phase locking (2PL) [3], read-one-write-all (ROWA) [4],

(C) 2012 Ohkawara et al.; licensee Springer. This is an Open Access article distributed under the terms of the Creative Commons Attribution License (http://creativecommons.org/licenses/by/2.0), which permits unrestricted use, distribution, and reproduction in any medium, provided the original work is properly cited. 
and quorum-based (QB) [5] protocols. In the 2PL protocol, all the replicas are first locked before they are read and write. On the other hand, only one replica is locked for read while every replica is locked for write in the ROWA protocol. In the QB protocol, subsets of the replicas for read and write operations are referred to as read quorum $Q_{r}$ and write quorum $Q_{w}$, respectively. Every pair of read and write quorums include at least one common replica. Only if every replica in a quorum could be locked, a transaction can manipulate the replicas in the quorum. In Cassandra [6], the synchronization scheme based on the quorum concept [4] is adopted.

Various types of objects including multimedia objects are distributed in P2P overlay networks. Multimedia objects are characterized in terms of quality of service (QoS) like frame rate and number of colours in addition to the contents. Thus, not only the content but also QoS parameters of an object are manipulated. For example, suppose there are three replicas $o_{1}, o_{2}$, and $o_{3}$ of a fully-coloured movie object $o$ in a quorum $Q$. A scene subobject is added to the replica $o_{2}$. On the other hand, the colour of another replica $o_{3}$ is changed with monochromatic one. The replica $o_{2}$ is newer than the replica $o_{3}$ in terms of the content while the replica $o_{3}$ is newer than the replica $o_{2}$ in terms of number of colours. Thus, replicas are partially ordered in terms of newness of not only content but also QoS parameters in a quorum. The partially ordering newness-precedent relation $\preceq$ among replicas of a multimedia object is defined in the paper [7]. On the other hand, replicas of a file object are totally ordered just in terms of newness of content in the traditional QB protocol. Here, there is no newest replica in the quorum $Q$. A complete quorum includes a newest replica. A newest replica should be a monochromatic replica with the scene subobjects in the quorum $Q$. The replicas $o_{2}$ and $o_{3}$ can be made the newest one by degrading colours and adding the scene subobjects, respectively. Thus, even if a quorum is not complete, some replica $o_{i}$ might be made the newest by applying operations with data held in other replicas $o_{i}$ in the quorum. An incomplete quorum which can be complete is referred to as completable. The replica $Q$ is completable. We discuss how to obtain the newest replica in an incomplete but completable quorum. In the traditional QB protocol, every quorum is complete. However, multimedia quorums can be completable.

We propose a multimedia quorum-based $(M Q B)$ protocol in this paper. Here, each replica of a multimedia object holds the vector of counters, where there is one counter for each of the content and QoS parameters. If a transaction issues a read operation $o p$, the transaction selects the newest replica $o_{i}$ in a quorum $Q_{o p}$. If not found, one replica $o_{i}$ is selected and is made newest by obtaining operations and data which are not performed on the replica $o_{i}$ through communicating with other replicas. Then, the transaction reads the replica $o_{i}$ in the quorum $Q_{o p}$. The content and QoS parameters of every replica are updated to be the newest. Here, computation and communication resources are consumed to update every replica in the quorum $Q_{o p}$. In order to reduce the computation and communication overheads, every quorum is tried to be completable, that is, only the counter vector of every replica is updated in the quorum $Q_{o p}$ but all the replicas themselves are not updated. We evaluate the MQB protocol compared with the QB protocol and show the communication overhead in the MQB protocol can be reduced with the QB protocol.

In section "Method", we discuss the newness-precedent relations on replicas of multimedia objects. In section "Evaluation", we discuss the multimedia quorum-based (MQB) protocol to maintain the mutually consistency of replicas. In section "Conclusions", 
we evaluate the MQB protocol compared with the QB protocol in terms of communication overheads.

\section{Method}

\section{Partially Ordering Relations of Multimedia Replicas Multimedia objects}

A multimedia object $o$ is characterized in terms of not only content parameter $o . c$ but also quality of service (QoS) parameters o.Q. A content $o . c$ shows data structure, i.e. the part_ of structure of subobjects. QoS $o . Q$ is specified in a tuple of QoS parameters $\left\langle q_{1}, \ldots, q_{l}\right\rangle$ $(l \geq 0)$. Frame rate and resolution are examples of QoS parameters. Thus, each replica $o_{i}$ of an object $o$ is specified in a pair of the content $o_{i} . c$ and QoS parameters $o_{i}$.Q. It is noted a traditional replica $o_{i}$ like a text object is just specified in terms of a content $o_{i}$.c. The content $o_{i} . c$ in a replica $o_{i}$ is manipulated in a content operation like delete-subobject while QoS parameters. $o_{i}$. $Q$ is manipulated in a $Q o S$ operation like change-colour.

In a QoS parameter frame rate $(f r), 40[\mathrm{fps}]$ is richer than $20[\mathrm{fps}]$. Thus, for a pair of values $x$ and $y$ of a QoS parameter $q_{k}, y$ is richer than $x(x \rightarrow y)(y$ is poorer than $x)$ iff $y$ includes more volume of data than $x$. For example, $20 \rightarrow 40$ [fps]. Let $c_{1}$ and $c_{2}$ be a pair of contents of an object $o$. A content parameter $c_{2}$ is richer than a content $c_{1}\left(c_{1}\right.$ is poorer than $\left.c_{2}\right)\left(c_{1} \rightarrow c_{2}\right)$ if $c_{1}$ is a component of $c_{2}$. A value $x$ can be obtained by just removing some data from a value $y$ if $x \rightarrow y$. However, if $y \rightarrow x$, the value $x$ cannot be obtained without adding any data to the value $y$. For example, a fully coloured movie object can be degraded to a monochromatic one by just removing the colour data. However, we have to add colour data to a monochromatic object in order to change with a coloured one.

A scheme of an object $o$ is written in a tuple $\left\langle p_{0}, p_{1}, \ldots, p_{l}\right\rangle$ where the first parameter $p_{0}$ stands for a content parameter $o . c$ and the $k$ th parameter $p_{k}$ indicates a QoS parameter $o . q_{k}(k=1, \ldots, l) . o . p_{i}$ shows a parameter $p_{i}$ of an object $o(i=0,1, \ldots, l)$.

\section{Newness-precedent relation}

Let $O$ be a set $\left\{o_{1}, \ldots, o_{n}\right\}$ of replicas of an object $o(n \geq 1)$ in the system. Here, the content parameter $o_{j} . c$ of a replica $o_{j}$ is newer than the content $o_{i} . c$ of another replica $o_{i}\left(o_{i} . c \prec o_{j} . c\right)$ iff (if and only if) the content parameter $o_{j} . c$ is updated, e.g. some subobject is deleted but $o_{i} \cdot c$ is not updated. A content $o_{i} . c$ precedes a content $o_{j} . c\left(o_{i} . c \preceq o_{j} . c\right)$ iff $o_{i} \cdot c \prec o_{j} . c$ or $o_{i} \cdot c=o_{j}$.c. A QoS parameter $o_{j} \cdot q_{k}$ of a replica $o_{j}$ is newer than $o_{i} \cdot q_{k}\left(o_{i} \cdot q_{k} \prec o_{j} \cdot q_{k}\right)$ iff the parameter $q_{k}$ is changed in the replica $o_{i}$ but is not in the replica $o_{j}$. For example, a monochromatic replica $o_{j}$ is obtained by changing the QoS parameter $\mathrm{cl}$ (colour) of a fully coloured replica $o_{i}$. Here, the QoS parameter $o_{j} . c l$ is newer than $o_{i} . c l\left(o_{i} . c l \prec o_{j} . c l\right)$. A QoS parameter $o_{i} \cdot q_{k}$ precedes $o_{j} \cdot q_{k}$ with respect to newness $\left(o_{i} \cdot q_{k} \preceq o_{j} \cdot q_{k}\right)$ iff $o_{i} \cdot q_{k} \prec o_{j} \cdot q_{k}$ or $o_{i} \cdot q_{k}=o_{j} \cdot q_{k}$.

Replicas in the replica set $O$ are partially ordered in the newness-precedent relation $\preceq$ $\left(\subseteq O^{2}\right)$. A replica $o_{i}$ precedes a replica $o_{j}$ with respect to newness $\left(o_{i} \preceq o_{j}\right)$ iff $o_{i} \cdot c \preceq o_{j} . c$ and $o_{i} \cdot q_{k} \preceq o_{j} \cdot q_{k}$ for every QoS parameter $q_{k}(k=0,1, \ldots, l)$. A replica $o_{i}$ is equivalent with a replica $o_{j}\left(o_{i} \equiv o_{j}\right)$ iff $o_{i} \cdot c=o_{j} \cdot c$ and $o_{i} \cdot q_{k}=o_{j} \cdot q_{k}$ for every QoS parameter $q_{k}$. A replica $o_{i}$ is newer than another replica $o_{j}\left(o_{i} \prec o_{j}\right)$ iff $o_{i} \preceq o_{j}$ but $o_{i} \not \equiv o_{j}$. A replica $o_{i}$ is uncomparable with a replica $o_{j}\left(o_{i} \mid o_{j}\right)$ iff neither $o_{i} \preceq o_{j}$ nor $o_{j} \preceq o_{i}$. In the traditional quorum-based $(\mathrm{QB})$ protocols [8] [3], replicas in the replica set $O$ are totally 
ordered, i.e. for every pair of replicas $o_{i}$ and $o_{j}$ in the replica set $O$, either $o_{i} \equiv o_{j}$ or $o_{j} \prec o_{i}$, that is, $o_{i} \preceq o_{j}$. On the other hand, replicas of a multimedia object $o$ are partially ordered in the newness-precedent relation $\preceq$. For example, the content parameter $o_{j} . c$ of a replica $o_{j}$ is newer than the content $o_{i} . c\left(o_{i} . c \prec o_{j} . c\right)$ while some QoS parameter $o_{i} \cdot q_{k}$ is newer than $o_{j} \cdot q_{k}\left(o_{j} \cdot q_{k} \prec o_{i} \cdot q_{k}\right)$. Here, a pair of replicas $o_{i}$ and $o_{j}$ are uncomparable $\left(o_{i} \mid o_{j}\right)$.

\section{Newest replica in a quorum}

In terms of the newness precedent relation $\preceq$, we define the least upper bound (lub) and greatest lower bound (glb) of replicas. $o_{i} \cup o_{j}$ shows a least upper bound (lub) of a pair of replicas $o_{i}$ and $o_{j}$, which is a replica $o_{k}$ such that $o_{i} \preceq o_{k}$ and $o_{j} \preceq o_{k}$ and there is no replica $o_{h}$ such that $o_{i} \preceq o_{h} \preceq o_{k}$ and $o_{j} \preceq o_{h} \preceq o_{k} . o_{i} \cap o_{j}$ indicates a greatest lower bound (glb) of replicas $o_{i}$ and $o_{j}$, which is a replica $o_{k}$ such that $o_{k} \preceq o_{j}$ and $o_{k} \preceq o_{j}$ and there is no replica $o_{h}$ such that $o_{k} \preceq o_{h} \preceq o_{i}$ and $o_{k} \preceq o_{h} \preceq o_{j}$. Let $Q$ be a quorum of replicas $o_{1}, \ldots, o_{n}$. $\cup Q$ indicates the lub $o_{1} \cup \ldots \cup o_{n}$ of every replica in the set $Q$, i.e. top replica of the set $Q$, which shows the newest replica in the quorum $Q$. A replica $o_{i}$ is maximal iff there is no replica $o_{j}$ in the quorum $Q$ such that $o_{i} \preceq o_{j}$. Max shows a subset of maximal replicas in the quorum $Q$. A quorum $Q$ is referred to as complete iff the lub $\cup Q$ exists in the quorum $Q$. Here, a quorum $Q$ is referred to as complete iff there is a top replica $\cup Q$ in the quorum $Q$.

Figure 1 shows a quorum $Q$ of five replicas $o_{1}, o_{2}, o_{3}, o_{4}$, and $o_{5}$ of a multimedia object $o, Q=\left\{o_{1}, o_{2}, o_{3}, o_{4}, o_{5}\right\}$. Each replica $o_{i}$ has a content parameter $(c)$ and QoS parameter colour $(c l)$, i.e. $o_{i}=\langle c, c l\rangle$. Here, a directed edge $o_{i} \rightarrow o_{j}$ shows the newness-precedent relation $o_{i} \preceq o_{j}$. A replica $o_{1}$ is composed of three fully coloured subobjects, blueberry $b$, orange $r$, and strawberry $s$. The content parameter $c$ is $\langle b, r, s\rangle, o_{1}=\langle\langle b, r, s\rangle$, fullycolour $(f c)\rangle$. Suppose initially $o_{1} \equiv o_{2} \equiv o_{3} \equiv o_{4} \equiv o_{5}$.

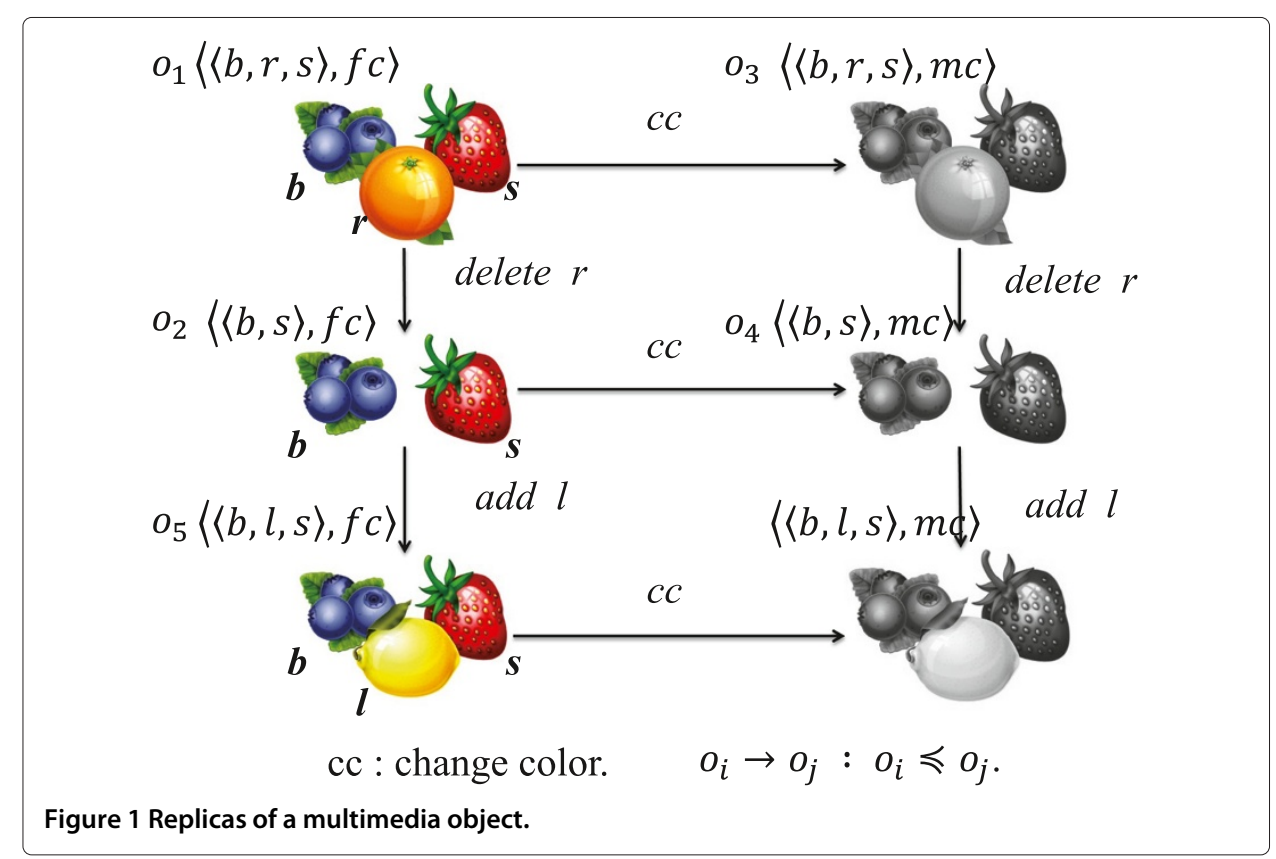


1 In a content operation delete, a subobject $r$ is removed in the replicas $o_{2}$ and $o_{5}$. Here, $o_{2} \equiv o_{5}=\langle\langle b, s\rangle, f c\rangle$. For a pair of the replicas $o_{2}$ and $o_{5}$, the content parameters $o_{2} . c$ and $o_{5} . c$ are newer than $o_{1} . c\left(o_{1} . c \preceq o_{2} . c\right)$ and $o_{1} . c \preceq o_{5} . c$ while the QoS parameters $o_{2} . c l$ are $o_{5} . c l$ are the same as $o_{1} . c l, o_{1} . c l=o_{2} . c l$. Hence, the replica $o_{1}$ precedes the replica $o_{2}\left(o_{1} \preceq o_{2}\right)$ and $o_{1} \preceq o_{5}$. Similarly, $\left\{o_{3}, o_{4}\right\} \preceq o_{2}$ and $\left\{o_{3}, o_{4}\right\}$ $\preceq o_{5} .\left\{o_{1}, o_{3}, o_{4}\right\} \preceq o_{5}$.

2 Next, a pair of the replicas $o_{3}$ and $o_{4}$ are changed by degrading with monochromatic (mc) ones by a down-colour (dc) operation; $o_{3} \equiv o_{4}=\langle\langle b, r, s\rangle, m c\rangle$. Here, the QoS parameters $c l$ of the replicas $o_{1}$ and $o_{2}$ are newer than $o_{1} . c l\left(o_{1} . c l \preceq o_{3} . c l\right)$ and $o_{1} . c l \preceq o_{4} \cdot c l$ while $o_{3} \cdot c=o_{4} \cdot c=o_{1} \cdot c=\langle b, r, s\rangle$. The replica $o_{1}$ precedes the replica $o_{3}\left(o_{1} \preceq o_{3}\right)$ and $o_{1} \preceq o_{3}$. Similarly, $o_{1} \preceq o_{4}$. Here, a pair of the replicas $o_{2}$ and $o_{3}$ are uncomparable $\left(o_{2} \mid o_{3}\right)$. Similarly, $o_{5}\left|o_{3}, o_{2}\right| o_{4}$, and $o_{5} \mid o_{4}$.

3 Then, the subobject orange $r$ is deleted in the replica $o_{4} ; o_{4}=\langle\langle b, s\rangle, m c\rangle$. Here, $o_{2} \cup o_{3}=o_{4}$.

4. A lemon subobject $l$ is added to the replica $o_{5}$. Here, $o_{5}=\langle\langle b, l, s\rangle, f c\rangle$. Here, there is no lub $o_{4} \cup o_{5}$ in the quorum $Q$. A pair of the replicas $o_{4}$ and $o_{5}$ are maximal and the replica $o_{1}$ is a bottom of the quorum Q, i.e. $o_{1}=o_{2} \cap o_{3}$ as shown in Figure 2. There is no top replica $\cup Q$ in the quorum $Q$. The lub $o_{4} \cup o_{5}$ shows the newest replica for the replicas in the quorum $Q$. By changing the colour $c l$ of the replica $o_{5}$ into $\mathrm{mc}$ or deleting the orange $r$ from the replica $o_{4}$, a top replica $\langle\langle b, s\rangle, m c\rangle\left(=o_{4} \cup o_{5}\right)$ can be obtained.

In Figure 2, the vertical axis shows the newness of the content parameter $c$. The content $c=\langle b, r, s\rangle$ is changed with the content $\langle b, s\rangle$, i.e. $\langle b, r, s\rangle \preceq\langle b, s\rangle$. Hence, $o_{1} . c(=\langle b, r, s\rangle)$ includes a larger volume of data than $o_{2} . c(=\langle b, s\rangle)$, i.e. $o_{1} . c \supseteq o_{2} . c$. The horizontal axis indicates the newness of the QoS parameter $c l$. The QoS parameter $c l$ is changed from $f c$ to $m c$, i.e. $f c \preceq m c$. The QoS parameter $o_{1} . c l(=f c)$ includes more volume of data than $o_{2} . c l(=m c)$. Thus, $\langle b, s\rangle \rightarrow\langle b, r, s\rangle$ and $m c \rightarrow f c$. A replica $o_{j}$ is richer than a replica $o_{i}\left(o_{i} \rightarrow o_{j}\right)$ iff $o_{i} \cdot c \rightarrow o_{j} . c$ and $o_{i} \cdot q_{k} \rightarrow o_{j} \cdot q_{k}$ for every QoS parameter $q_{k}$. This means, the replica $o_{i}$ can be obtained by deleting data and degrading the richer replica $o_{j}$ to a less QoS one.

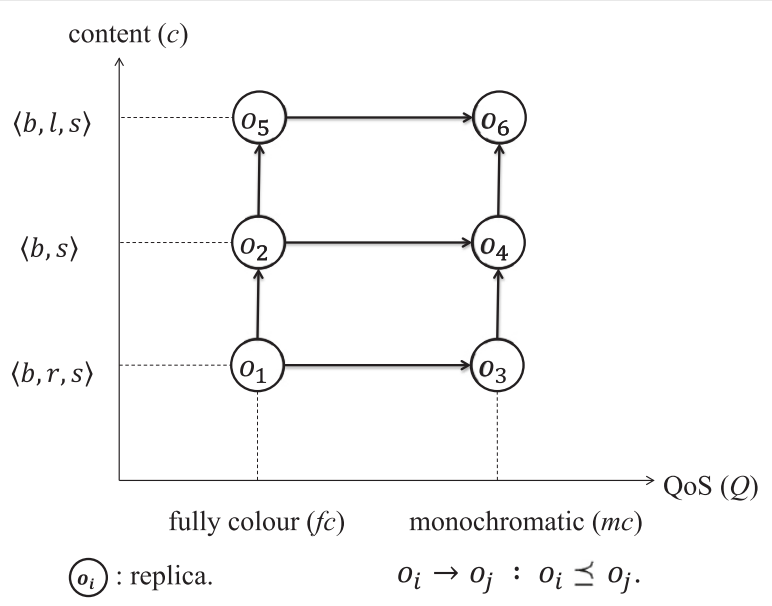

Figure 2 Newness precedence of content and QoS. 
In the $\mathrm{QB}$ protocol [9], there is at least one newest replica $o_{i}$ in a read quorum $Q_{r} . \mathrm{A}$ transaction reads the newest replica $o_{i}$ in the quorum $Q_{r}$.A transaction writes every replica in a write quorum $Q_{w}$. Then, every replica in the quorum $Q_{w}$ gets the newest. In the QB protocol, each replica has the version counter. The version counter of every replica in a write quorum $Q_{w}$ is incremented so that the version counter of each replica in the quorum $Q_{w}$ shows the maximum value in the replica set $O$. Hence, the write quorum $Q_{w}$ includes at least one newest replica whose version counter is the maximum. A replica whose version counter is the maximum is the newest. Every replica is required to be complete in the QB protocol.

For a pair of values $x$ and $y, \max (x, y)$ is defined to be the value $x$ if $y \preceq x$. Here, $\max (x, y)$ $=\max (y, x) \cdot \max (x, y)=\perp$ if $x \mid y$. The upgrade operation $o_{i}+o_{j}$ on a pair of replicas $o_{i}$ and $o_{j}$ a replica $o_{h}$ such that $o_{h} \cdot c=\max \left(o_{i} . c, o_{j} . c\right)$ and $o_{h} \cdot q_{k}=\max \left(o_{i} \cdot q_{k}, o_{j} \cdot q_{k}\right)$ for every QoS parameter $q_{k} .+Q$ shows $o_{1}+\ldots+o_{n}$ for a quorum $Q\left(=\left\{o_{1}, \ldots, o_{n}\right\}\right) .+Q$ shows a replica $o$ which may not be in a quorum $Q$ but which can be the top replica $\cup Q$. Here, let $m_{c}$ be $\max \left\{o_{i} . c \mid o_{i} \in Q\right\}$ and $m_{q_{k}}$ be $\max \left\{o_{i} . q_{k} \mid o_{i} \in Q\right\}$ in a quorum $Q$. A replica $o_{i}$ can be upgraded to an lub of a quorum Q if the content parameter $o_{i} . c$ and every QoS parameter $o_{i} . q_{k}$ could be changed to $m_{c}$ and $m_{q_{k}}$, respectively. In order to reduce the overhead to upgrade a replica, one of the maximal replicas is taken. For example, a maximal replica $o_{i}$ with the smallest number of parameters to be changed is taken. Then, the maximal replica $o_{i}$ is upgraded. A quorum $Q$ is referred to as completable iff $+Q$ is $\cup Q$. That is, some replica $o_{i}$ can be upgraded to the top replica $\cup Q$. The quorum $Q$ shown in Figure 1 is incomplete but completable since one of the maximal replicas $o_{5}$ and $o_{6}$ can be upgraded.

\section{Types of operations}

Let $o p$ be an operation supported by an object $o$, i.e. read or write operation.Let $Q_{o p}(\subseteq O)$ be a quorum for an operation $o p$. Here, there might not be the newest, i.e. top replica in the quorum $Q_{o p}$. That is, the lub $\cup Q_{o p}$ is not in the quorum $Q_{o p}$. Even if there is no top replica in the quorum $Q_{o p}$, there is some maximal replica $o_{i}$ in the quorum $Q_{o p}$.

There are two types of write operations by which replicas are changed:

1 Enriching $(E)$ type.

2 Impoverishing (I) type.

Suppose a value $x$ is changed with another value $y$ of a content or QoS parameter in an operation $o p$. Here, the value $x$ precedes the value $y$, i.e. $y$ is newer than $x(x \preceq y)$. If op is an enriching type of operation, $y$ is richer than $x(x \rightarrow y)$. Otherwise, $y \rightarrow x$. A richer replica $o_{i}$ can be easily changed into a poorer replica because data in the replica is just removed without using additional data not in the replica $o_{i}$. On the other hand, we need additional data which is not in a replica $o_{i}$ to change a poorer replica $o_{i}$ in order to a richer one. Thus, in an enriching operation, some volume of data is added to a replica $o_{i}$, i.e. the replica $o_{i}$ is enriched. For example, an orange subobject $r$ is added to the replica $o_{4}$ by a content operation insert as shown in Figure 2. The number of colours $(c l)$ is increased in a QoS operation up-colour $(u c)$, i.e. changed with the fully coloured one as shown in Figure 3 (a). This is an enriching operation. On the other hand, some data is removed from a replica in an impoverishing operation, i.e. the replica is made poorer. For example, some subobject, say an orange $r$ is deleted from a replica $o$ by a content operation delete as shown in Figure 3 (b). On the other hand, further data which is not in the replica is 


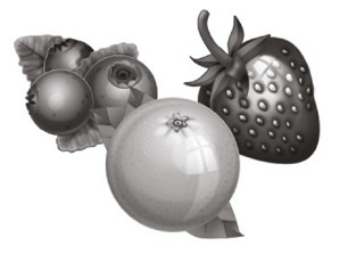

monochromatic $(m c)$

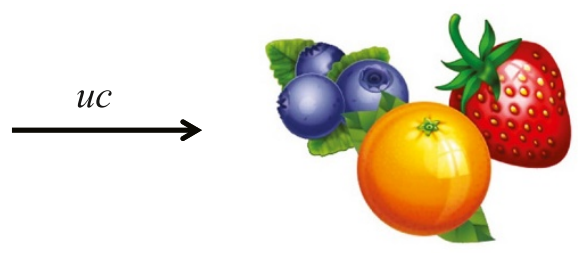

fully coloured $(f c)$

(a) Enriching operation.
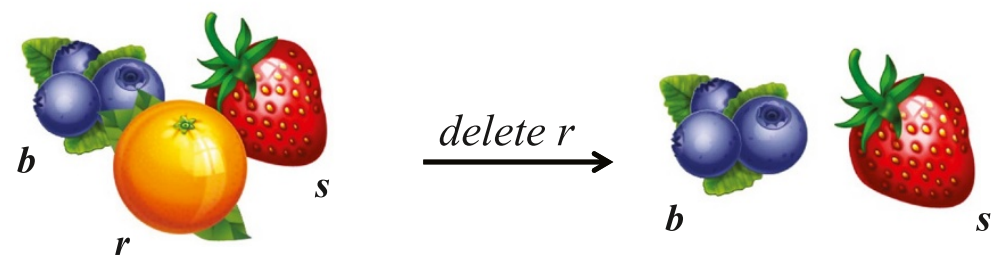

(b) Impoverishing operation.

Figure 3 Enriching and impoverishing operations.

required to increase the frame rate. Thus, it is easier to perform the impoverishing type of write operation than the enriching type on a replica.

Suppose there are a pair of monochromatic replicas $o_{i}$ and $o_{j}$ of a multimedia object $o$, which are composed of a blueberry $b$, orange $r$, and strawberry $s$ subobjects. A pair of the replicas $o_{i}$ and $o_{j}$ are equivalent, $o_{i} \equiv o_{j}$ where $o_{i} \cdot c=o_{j} \cdot c=\langle b, r, s\rangle$ and $o_{i} \cdot c l=o_{j} . c l=$ $m c$. Then, a transaction $T_{1}$ deletes an orange subobject $r$ in the replica $o_{i}$. The top, i.e. newest replica is the replica $o_{i}$ while $o_{j}$ is obsolete. Since delete is an impoverishing write operation, the replica $o_{j}$ can be made a newest one by just deleting the subobject $r$. On the other hand, suppose a transaction $T_{2}$ changes the colour $(\mathrm{cl})$ parameter of the replica $o_{i}$ to be fully coloured in an up-colour $(u c)$ operation. The $u c$ operation is an enriching one. In order to change the replica $o_{j}$, data to make the replica $o_{j}$ fully coloured has to be sent to the replica $o_{j}$ since the replica $o_{j}$ does not have the data while $o_{i}$ has the data. Even if the operation $u c$ which is applied to the replica $o_{i}$ is obtained to the replica $o_{j}$, the replica $o_{j}$ cannot be changed without obtaining the colour data from the newest replica $o_{i}$.

In the QB protocol, $Q_{o p_{i}} \cap Q_{o p_{j}} \neq \phi$ for every pair of quorums $Q_{o p_{i}}$ and $Q_{o p_{j}}$ of conflicting operations $o p_{i}$ and $o p_{j}$. The quorum-based protocol for abstract types of operations on objects is discussed in the paper [5].

\section{Multimedia Quorum-Based (MQB) Protocol}

\section{Counter vectors}

Let $Q$ be a quorum of replicas $o_{1}, \ldots, o_{n}(n \geq 1)$ of a multimedia object $o$. Each replica $o_{i}$ is characterized in terms of the content $o_{i} \cdot c$ and QoS parameters $o_{i} \cdot q_{1}, \ldots, o_{i} \cdot q_{l}(i=$ $1, \ldots, n)$. Here, a tuple of parameters $\left\langle p_{0}, p_{1}, \ldots, p_{l}\right\rangle$ is a scheme of a replica $o_{i}$. Here, the object $o_{i}$ is written as a tuple $\left\langle v_{0}, v_{1}, \ldots, v_{l}\right\rangle(l \geq 1)$ of values, where $v_{0}$ shows the content $c$ and $v_{k}$ stands for a value of a QoS parameter $q_{k}$ for $k=1, \ldots, l$. A vector $o_{i} . V$ $=\left\langle v c_{o}, v c_{1}, \ldots, v c_{l}\right\rangle$ of version counters is assigned to a replica $o_{i}=\left\langle o_{i} \cdot v_{0}, o_{i} \cdot v_{1}, \ldots, o_{i} \cdot v_{l}\right\rangle$. 
Initially, $o_{i} . V=\langle 0,0, \ldots, 0\rangle$. Each time an element $o_{i} . q_{k}$ is changed $(k=0,1, \ldots, l)$, the counter $o_{i} . v c_{k}$ is incremented so that the counter $o_{i} \cdot v c_{k}$ is the maximum in the quorum $Q$.

Suppose a counter $v c_{k}$ is incremented since a parameter $o_{i} \cdot p_{k}$ is changed in a replica $o_{i}$ by performing an operation $o p$ on the replica $o_{i}(k \in\{0,1, \ldots, l\})$. First, the maximum counter value $v$ is taken in a set $\left\{o_{i} . v c_{k} \mid o_{i} \in Q_{o p}\right\}$ of the counter values of the quorum $Q_{o p}$. In the quorum $Q_{o p}$, the maximum value $v$ of the counter $v c_{k}$ is incremented by one, $v=v$ +1 . Then, $o_{i} . v c_{k}=v$ on every replica $o_{i}$ in the quorum $Q_{o p}$. The value of the $k$ th parameter $o_{i} . q_{k}$ of a replica $o_{i}$ is the newest if the counter $v c_{k}$ is maximum in the quorum $Q_{o p}$.

Let us consider a quorum $Q$ of five replicas $o_{1}, o_{2}, o_{3}, o_{4}$ and $o_{5}$ shown in Figure 1 , $Q=\left\{o_{1}, o_{2}, o_{3}, o_{4}, o_{5}\right\}$. Initially, every replica is equivalent in the quorum $Q$, i.e. $o_{1} \equiv o_{2} \equiv o_{3} \equiv o_{4} \equiv o_{5}$. Each replica $o_{i}$ has one QoS parameter colour $(c l)$ and a vector $o_{i} . V=\left\langle o_{i} . v c_{0}, o_{i} . v c_{1}\right\rangle(i=1, \ldots, 5)$. In each vector $o_{i} . V, o_{i} . v c_{0}$ is a counter for the content parameter $o_{i} . c\left(=o_{i} . v_{0}\right)$ and $o_{i} . v c_{1}$ is a counter for the QoS parameter $c l$ (number of colours) $o_{i} . c l\left(=o_{i} . v_{1}\right)$. In every replica $o_{i}$, initially $o_{i} . V=\langle 0,0\rangle$ where $o_{i}$ is composed of three fully coloured subobjects $s, g$, and $a$, i.e. $o_{i}=\langle\langle s, g, a\rangle, f l\rangle$.

1 First, the fully coloured replicas $o_{2}$ and $o_{5}$ are updated by changing the colour parameter $c l$ with the monochromatic one, $o_{2}=o_{5}=\langle\langle s, g, a\rangle, m c\rangle$. The counter $v c_{1}$ is incremented by one. Here, $o_{2} . V=o_{5} . V=\langle 0,1\rangle$ since the second parameter $c l$ is changed.

2 Next, a pair of replicas $o_{3}$ and $o_{4}$ are obtained by deleting an orange subobject $r$, i.e. $o_{3} . V=o_{4} . V=\langle 1,0\rangle$ where $o_{3}=o_{4}=\langle\langle s, g\rangle, f c\rangle$ since the first content $v_{0}$ is changed.

3 Then, the orange subobject $r$ is deleted in the replica $o_{4}, o_{4}=\langle\langle s, g\rangle, m c\rangle$.

Here, $o_{4} . V=\langle 1,1\rangle$. Here, $o_{1} \preceq o_{2} \preceq o_{4}$ where $o_{1} . V \leq o_{2} . V$ and $o_{2} . V \leq o_{4} . V$.

4 Then, the fully coloured lemon subobject $l$ is added

to the replica $o_{5}, o_{5}=\langle\langle b, l, s\rangle, f c\rangle$. The counter vector $o_{5} . V$ is changed with $\langle 2,0\rangle$.

Here, a pair of the replicas $o_{2}$ and $o_{3}$ are uncomparable $\left(\begin{array}{lll}o_{2} & o_{3}\end{array}\right)$ where a pair of the vectors $o_{2} \cdot V=\langle 1,0\rangle$ and $o_{3} \cdot V=\langle 0,1\rangle$ are not comparable. $o_{1} \preceq o_{3}$ since $o_{1} . V<o_{3} . V . o_{3} \preceq o_{4}$ and $o_{3} \preceq o_{5}$ where $o_{3} \cdot V \leq o_{4} \cdot V$ and $o_{3} . V \leq o_{5} \cdot V, o_{4} \mid o_{5}$ since $o_{4} . V=\langle 1,1\rangle$ and $o_{5} \cdot V=\langle 2,0\rangle$. Figure 4 shows the vector $o_{i} . V$ of each replica

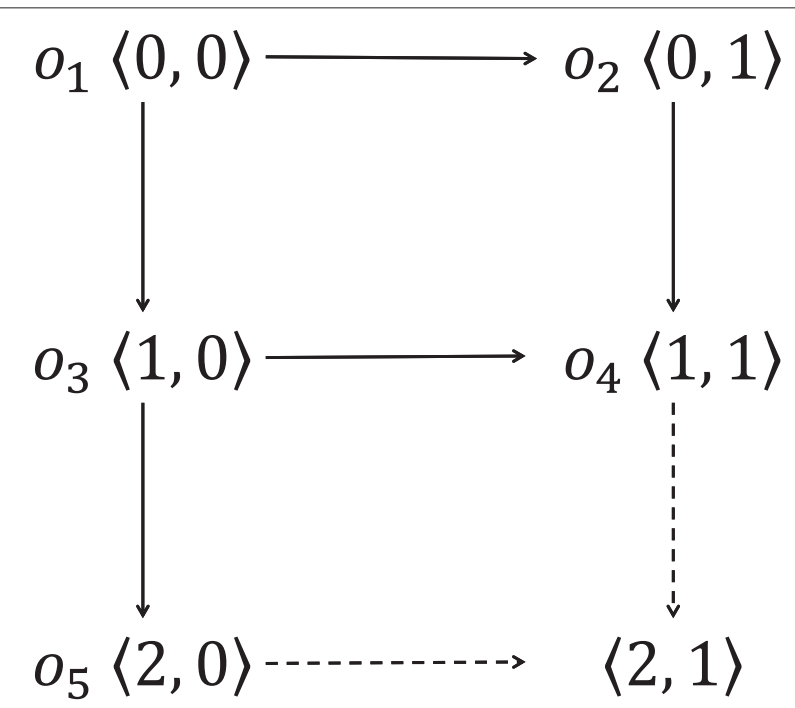

Figure 4 Counter vectors of replicas. 
$o_{i}(i=1, \ldots, 5)$. Here, a directed edge $o_{i} \rightarrow o_{j}$ shows the newness-precedent relation $o_{i} \preceq o_{j}$. Here, there is no top replica in the quorum $Q$. Here, $\max \left(o_{4} . v c_{0}, o_{5} . v c_{0}\right)=2$ and $\max \left(o_{4} . v c_{1}, o_{5} . v c_{1}\right)=1$. Hence, if the colour parameter $o_{5} . c l$ of the replica $o_{5}$ is changed with monochromatic one $m c$, the replica $o_{5}$ gets the top replica $\langle\langle b, l, s\rangle, m c\rangle$. Here, the counter $o_{5} . v c_{0}$ is incremented by one, $o_{5} . V=\langle 2,1\rangle$. In another way, the replica $o_{4}$ can be the newest replica $\langle\langle b, l, s\rangle, m c\rangle$ by adding a monochromatic lemon $l$ to the replica $o_{4}$.

\section{Write operations}

Suppose a transaction $T$ issues a write operation op to change the $k$ th parameter $p_{k}$ of a replica $o_{i}(0 \leq k \leq l)$. A transaction $T$ first locks a replica $o_{i}$ in the op mode before performing an operation $o p$ on the replica $o_{i}$. Here, a lock mode $o p_{1}$ is referred to as conflict with a lock mode $o p_{2}$ if an operation $o p_{1}$ conflicts with an operation $o p_{2}$. If the replica $o_{i}$ is already locked in a mode conflicting with the operation $o p$, the transaction $T$ has to wait.

\section{[Write procedure]}

1 First, the transaction $T$ locks every replica $o_{i}$ with an op mode in the quorum $Q_{o p}$. If every replica $o_{i}$ could not be locked, the transaction $T$ waits.

2 If successfully locked, the transaction $T$ writes the $k$ th element $o_{i} \cdot v_{k}$ of every replica $o_{i}$ and collects the vector $o_{i} . V$ from every replica $o_{i}$ in the quorum $Q_{o p}$.

$3 v c=\max \left(o_{1} . v c_{k}, \ldots, o_{n} . v c_{k}\right)$ and the transaction $T$ changes $o_{i} . v c_{k}$ with $v c+1$ in every replica $o_{i}$ of the quorum $Q_{o p}$.

The version counter $v c_{k}$ of every replica in the quorum $Q_{o p}$ is changed with the maximum value $v c$. In order to reduce the computation and communication overheads, the parameter $o_{i} \cdot p_{k}$ of every replica $o_{i}$ is not always changed while the counter is updated:

1 If the operation op is an enriching type, the parameter $o_{i} \cdot q_{k}$ of every replica $o_{i}$ is updated in the quorum $Q_{o p}$.

2 If $o p$ is an impoverishing type, the parameter $o_{i} \cdot q_{k}$ of only the top replica $o_{i}$ is updated.

We consider the replicas of the object $o$ shown in Figure 5. Suppose a transaction $T_{1}$ adds a lemon subobject $l$ to the replicas. The operation $a d d$ is an enriching type of write operation. Suppose $Q_{a d d}$ is a quorum $\left\{o_{1}, o_{2}, o_{3}\right\}$ of the replicas for the add operation. Here, $o_{1}=\langle\langle b, r, s\rangle, f c\rangle, o_{2}=\langle\langle b, s\rangle, f c\rangle$, and $o_{3}=\langle\langle b, r, s\rangle, m c\rangle$. A pair of the replicas $o_{2}$ and $o_{3}$ are maximal, $\operatorname{Max}_{Q_{\text {add }}}=\left\{o_{2}, o_{3}\right\} \subseteq Q_{\text {add }}$. Here, $\max \left(o_{1} . c, o_{2} . c, o_{3} . c\right)=\langle b, s\rangle$

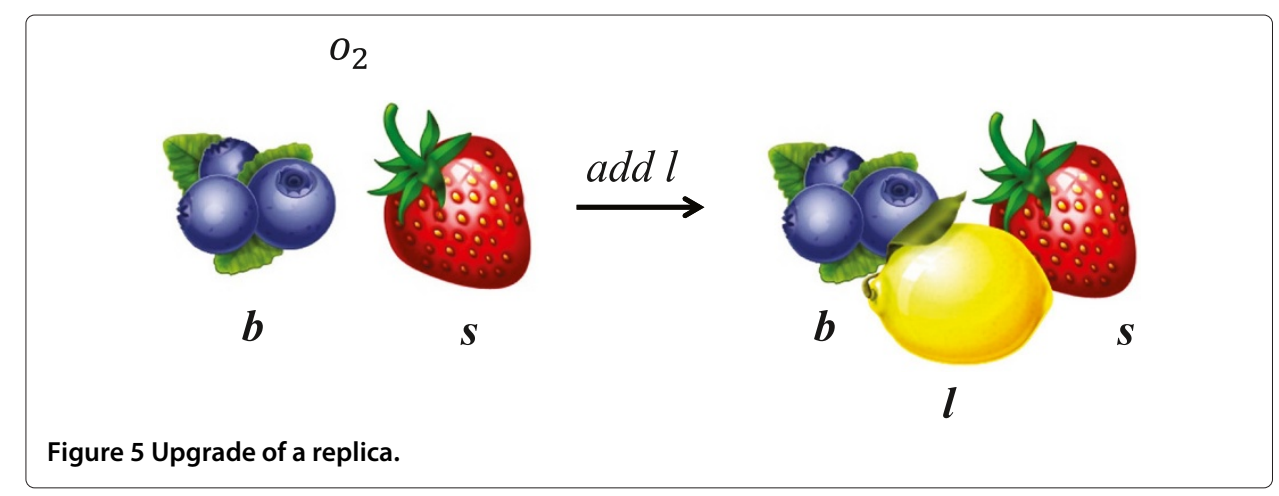


$\left(=o_{2} \cdot c=o_{3} \cdot c\right)$ and $\max \left(o_{1} . c l, o_{2} . c l, o_{3} . c l\right)=m c$. Here, the replica $o_{2}$ can be the top replica $\langle\langle b, s\rangle, m c\rangle\rangle$ by upgrading the replica $o_{2}, o_{2}=+Q_{a d d}$. Then, the lemon subobject $l$ is added to the replica $o_{2}$. Here, $o_{2}=\langle\langle b, l, s\rangle, m c\rangle$. A pair of the other replicas $o_{1}$ and $o_{3}$ are changed so that $o_{1} \equiv o_{2}$ and $o_{3} \equiv o_{2}$. That is, the colour parameter $c l$ of the replica $o_{1}$ is changed with monochromatic one $m c$, the orange subobject $r$ is deleted, and the lemon $l$ is added to the replica $o_{1}$. The orange $r$ is deleted and the lemon $l$ is added to the replica $o_{3}$. Then, a pair of the replicas $o_{2}$ and $o_{3}$ get equivalent with the replica $o_{1}\left(o_{2} \equiv o_{3} \equiv o_{1}=\right.$ $\langle\langle b, s\rangle, m c\rangle)$.

Next, suppose a transaction $T_{2}$ deletes a subobject $b$. Here, suppose there are three replicas $o_{1}, o_{2}$, and $o_{3}$ shown in Figure 2. One maximal replica $o_{2}$ is taken and upgraded to $\langle\langle b, s\rangle, m c\rangle$. Then, $b$ is removed. Here, $o_{2}=\langle\langle s\rangle, m c\rangle$. Since the delete operation is an impoverishing one, the other replicas $o_{1}$ and $o_{2}$ are not updated and the delete operation is logged in $o_{1}$ and $o_{2}$.

\section{Read operations}

Next, suppose a transaction $T$ issues a operation $o p$ to replicas of an object $o$ to read the parameter $p_{k}(k=0,1, \ldots, l)$. The transaction $T$ has to read the $k$ th parameter $o_{i} \cdot p_{k}$ of the newest replicas $o_{i}$ in the quorum $Q_{o p}$. In order to read the newest replica $o_{i}$, the transaction $T$ has to read the version counter $o_{j} . v c_{k}$ from every replica $o_{i}$ in the quorum $Q_{o p}$ :

\section{[Read procedure]}

1 First, the transaction $T$ locks every replica with an op mode in the quorum $Q_{o p}$. If every replica could not be locked, the transaction $T$ waits.

2 If every replica could be successfully locked, the transaction $T$ collects the vector $o_{i} . V$ from every replica $o_{i}$ in the quorum $Q_{o p}$.

3 If there is a replica $o_{i}$ such that $o_{j} . V \leq o_{i} . V$ for every replica $o_{j}$ in the quorum $Q_{o p}$, the replica $o_{i}$ is the top of the replicas in the quorum $Q_{o p}$, i.e. $o_{i}=\cup Q_{o p}$ and is the newest in the quorum $Q_{o p}$. The transaction $T$ reads the replica $o_{i}$ and change the counter $o_{i} . v c_{k}$ in every replica in the quorum $Q_{o p}$.

4 If there is no top replica in the quorum $Q_{o p}$, the transaction $T$ has to upgrade one maximal replica $o_{i}$ in $Q_{o p}$, i.e. $o_{i}=+Q_{o p}$ which is the top $\cup Q_{o p}$ of the quorum $Q_{o p}$. The transaction $T$ reads the $k$ th element $o_{i} . v_{k}$ of the replica $o_{i}$ and changes the counter $o_{j} . v c_{k}$ with $o_{i} . v c_{h}$ in every replica in $Q_{o p}$.

\section{Upgrade of a maximal replica}

If a top replica is not in the quorum $Q_{o p}$, the transaction $T$ has to obtain a top replica from the replicas in the quorum $Q_{o p}$ in the read procedure. We discuss how to upgrade a maximal replica $o_{i}$ to the top replica, i.e. $o_{i}=+Q_{o p}$ by using the vectors of the replicas. A replica $o_{i}$ is referred to as satisfy a counter vector $V=\left\langle v c_{0}, v c_{1}, \ldots, v c_{l}\right\rangle$ iff $o_{i} . v c_{k}=v c_{k}$ for $k=0,1, \ldots, l$.

First, one maximal replica $o_{i}$ is selected in the quorum $Q_{o p}$ as follows:

\section{[Selection of a maximal replica]}

1 The transaction $T$ obtains a vector $V=\left\langle v c_{0}, v c_{1}, \ldots, v c_{l}\right\rangle$ where $v c_{k}=$ $\max \left(\left\{o_{i} . v c_{k} \mid o_{i} \in Q_{o p}\right\}\right)$ for $k=0,1, \ldots, l$ from the collection of the vectors collected in the quorum $Q_{o p}$. 
2 A replica $o_{i}$ which satisfies the vector $V=\left\langle v c_{0}, v c_{1}, \ldots, v c_{l}\right\rangle$, i.e. $o_{i} . v c_{k}=v c_{k}$ for every $k=0,1, \ldots, l$, shows the top replica $\cup Q_{o p}$. If the replica $o_{i}$ is found, the transaction $T$ reads the replica $o_{i}$.

3 Otherwise, the transaction $T$ selects a replica $o_{i}$ where $\mid\left\{v c_{k} \mid o_{i} \cdot v c_{k}=v c_{k}\right.$ for $k=$ $0,1, \ldots, l\} \mid$ is the maximal in the quorum $Q_{o p}$. That is, a maximal replica $o_{i}$ is selected so that the overhead to change the replica can be reduced.

The replica $o_{i}$ found at step 3 is not the top in the quorum $Q_{o p}$. Here, a parameter $p_{k}$ of a replica $o_{i}$ is current if $o_{i} \cdot v c_{k}$ is maximum. Otherwise, the parameter $p_{k}$ is obsolete. Hence, the transaction $T$ updates the parameters of the replica $o_{i}$ as follows:

\section{[Upgrade of a maximal replica]}

1 For each obsolete parameter $p_{k}$, the transaction $T$ finds a replica $o_{j}$ where $o_{j} \cdot p_{k}$ is current in the quorum $Q_{o p}$ which satisfies $o_{i} . v c_{k}<v c_{k}$.

2 The transaction $T$ updates each obsolete parameter $o_{i} \cdot p_{k}$ with the current one $o_{j} . p_{k}$ by using the replica $o_{j}$.

3 The vector $o_{i} . V$ is updated as $o_{i} . V=V$.

At step 1, the replica $o_{j}$ found by the transaction $T$ has the newest value of each obsolete parameter $p_{k}$ of the replica $o_{i}$. The value of the parameter $o_{i} \cdot p_{k}$ has to be enriched to the parameter value $o_{j} . p_{k}$. If the parameter value $o_{i} \cdot p_{k}$ is richer than $o_{j} \cdot p_{k}$, i.e. $o_{j} \cdot p_{k} \rightarrow o_{i} \cdot p_{k}$, the QoS parameter of the replica $o_{i}$ can be impoverished just by deleting some data in the replica $o_{i}$ without using additional data. Otherwise, the parameter $p_{k}$ of the replica $o_{i}$ has to be enriched, i.e. we need further data which is not in the replica $o_{i}$ to enrich the value of the QoS parameter $p_{k}$. Hence, the content $o_{i} \cdot v_{0}$ of the replica $o_{i}$ is required to be the same as the replica $o_{j}$, i.e. $o_{i} \cdot v_{0}=o_{j} \cdot v_{0}$.

The elements $o_{i} . v_{0}, o_{i} . v_{1}, \ldots, o_{i} . v_{l}$ of every replica $o_{i}$ in the quorum $Q_{o p}(\subseteq O)$ are changed with the newest ones. In addition, the vector $o_{i} . V$ of every replica $o_{i}$ in the quorum $Q_{o p}$ has to be changed to be larger than every replica in the replica set $O$. In every read operation $o p^{\prime}$, every replica in the quorum $Q_{o p^{\prime}}$ is changed with a replica equivalent with the top replica. It is sure at least one top replica of the replica set $O$ is included in the quorum $Q_{o p^{\prime}}$. However, the overhead to change every replica in the quorum $Q_{o p^{\prime}}$ is increased. Suppose one top replica is read by the operation $o p^{\prime}$ and other replicas in the quorum $Q_{o p^{\prime}}$ are not changed. Hence, the quorum $Q_{o p}$ may not include the top replica. Here, the content and QoS parameters of every replica in the quorum $Q_{o p}$ can be changed since they are just overwritten. However, the maximum vector value obtained by all the replicas in the quorum $Q_{o p}$ may not be the maximum in the replica set $O$. Suppose $Q_{o p^{\prime}}=\left\{o_{1}, o_{2}, o_{3}\right\}$ and $Q_{o p}=\left\{o_{3}, o_{4}\right\}$. Here, suppose the replica $o_{1}$ is the top replica. A transaction $T_{1}$ reads the top replica $o_{1}$ in a read operation $o p$ ' but does not change the other replicas $o_{2}$ and $o_{3}$. Then, another transaction $T_{2}$ writes the replicas $o_{3}$ and $o_{4}$ in a write-type operation op. Here, the vectors of replicas $o_{3}$ and $o_{4}$ are not the newest while the vector of the replica $o_{1}$ is the newest.

If every replica is updated in a read operation, it implies larger communication and computation overhead to bring update data to every replica and then update every replica in the read quorum. In order to reduce the overhead, we take the following approach: 


\section{[Completable quorum]}

- In a read operation op, only the vector $o_{i} . V$ of every replica $o_{i}$ except the top replica is changed but the content $o_{i} \cdot v_{0}$ and QoS parameters $o_{i}$.Q of the replica $o_{i}$ are not changed.

In a write operation $o p$, the vector $V$ which shows the top replica in the replica set $O$ can be obtained in the quorum $Q_{o p}$. In the example of the quorums $Q_{o p^{\prime}}$ and $Q_{o p}$, the vectors of the replicas $o_{1}, o_{2}$, and $o_{3}$ are updated while the content and QoS parameters of the replicas $o_{2}$ and $o_{3}$ are not updated by the transaction $T_{1}$. Then, the transaction $T_{2}$ overwrites every replica in the quorum $Q_{o p}$. Here, the vector $o_{3} . V$ of the replica $o_{3}$ is the newest since $o_{3} . V$ is updated by the transaction $T_{1}$. Hence, the vector of the replica $o_{3}$ is incremented and then the vector $o_{4} . V$ of the replica $o_{4}$ is changed with $o_{3} . V$.

\section{Evaluation}

We would like to evaluate the multimedia quorum-based (MQB) protocol compared with the traditional quorum-based $(\mathrm{QB})$ protocol in terms of communication overhead. In the $\mathrm{MQB}$ protocol, if a transaction issues a read operation, every replica in a read quorum $Q_{r}$ is not updated while the vector of every replica is updated. We show how much the communication overhead to update every replica in the quorum $Q_{r}$ can be reduced in the MQB protocol.

Suppose there are $n$ replicas, $o_{1}, \ldots, o_{n}(n \geq 1)$ of an object $o$. Suppose there are two types of operations, read $(r)$ and write $(w) . Q_{r}$ and $Q_{w}$ show a pair of read and write quorums, respectively. $n_{r}$ shows the number $\left|Q_{r}\right|$ of replicas in the quorum $Q_{r}$ and $n_{w}=\left|Q_{w}\right|$. Let $f_{r}$ and $f_{w}$ be a pair of probability that a replica is included in the quorums $Q_{r}$ and $Q_{w}$, respectively. We assume each quorum is randomly constructed. That is, $f_{r}=n_{r} / n$ and $f_{w}=n_{w} / n$. According to the quorum properties, $f_{r}+f_{w}>1$ and $f_{w}>0.5$. Let $f$ be $f_{r}+f_{w}-1$. Here, $f$ shows probability that a replica is included in both the quorums $Q_{r}$ and $Q_{w} . f>0$.

In the $\mathrm{QB}$ protocol, a transaction $T$ first issues a lock request to every replica in a quorum $Q_{o p}$ to perform an operation $o p \in\{r, w\}$. If every replica is successfully locked in the quorum $Q_{o p}$, the transaction $T$ issues an operation op to replicas in $Q_{o p}$. First, suppose the transaction $T$ issues a write op to every replica in the write quorum $Q_{w}$ and updates the version counter of every replica. Here, totally $4 \cdot n_{w}\left(=4 \cdot n \cdot f_{r}\right)$ messages are transmitted. In order to write replicas, data is sent to every replica in the write quorum $Q_{w}$. Let $d$ be the size of the update data, e.g. the size of a replica. The expected volume of data transmitted is $n \cdot f_{w} \cdot d$.

On the other hand, the transaction $T$ issues a read operation $o p$ to one replica and receives a value of the replica in the read quorum $Q_{r}$. Then, the transaction $T$ sends the newest value to every other replica and updates the version vector of every replica in the QB protocol. The totally $4 \cdot n_{r}$ messages are transmitted between the transaction $T$ and the replicas. In the $\mathrm{QB}$ protocol, the newest value of the replicas in the quorum $Q_{r}$ is read into the transaction $T$ and is transmitted to every other replica which is in the quorum $Q_{r}$ but not in the quorum $Q_{w}$. Hence, the expected volume of data transmitted is $n \cdot f_{r} \cdot\left(1-f_{w}\right) \cdot d$.

In the MQB protocol, the transaction $T$ reads the top replica and updates the version counter of every replica in the read quorum $Q_{r}$. However, the other replicas are not updated in the quorum $Q_{r}$. The number $4 \cdot n_{r}\left(=4 \cdot n \cdot f_{w}\right)$ and $4 \cdot n_{w}\left(=4 \cdot n \cdot f_{r}\right)$ of 


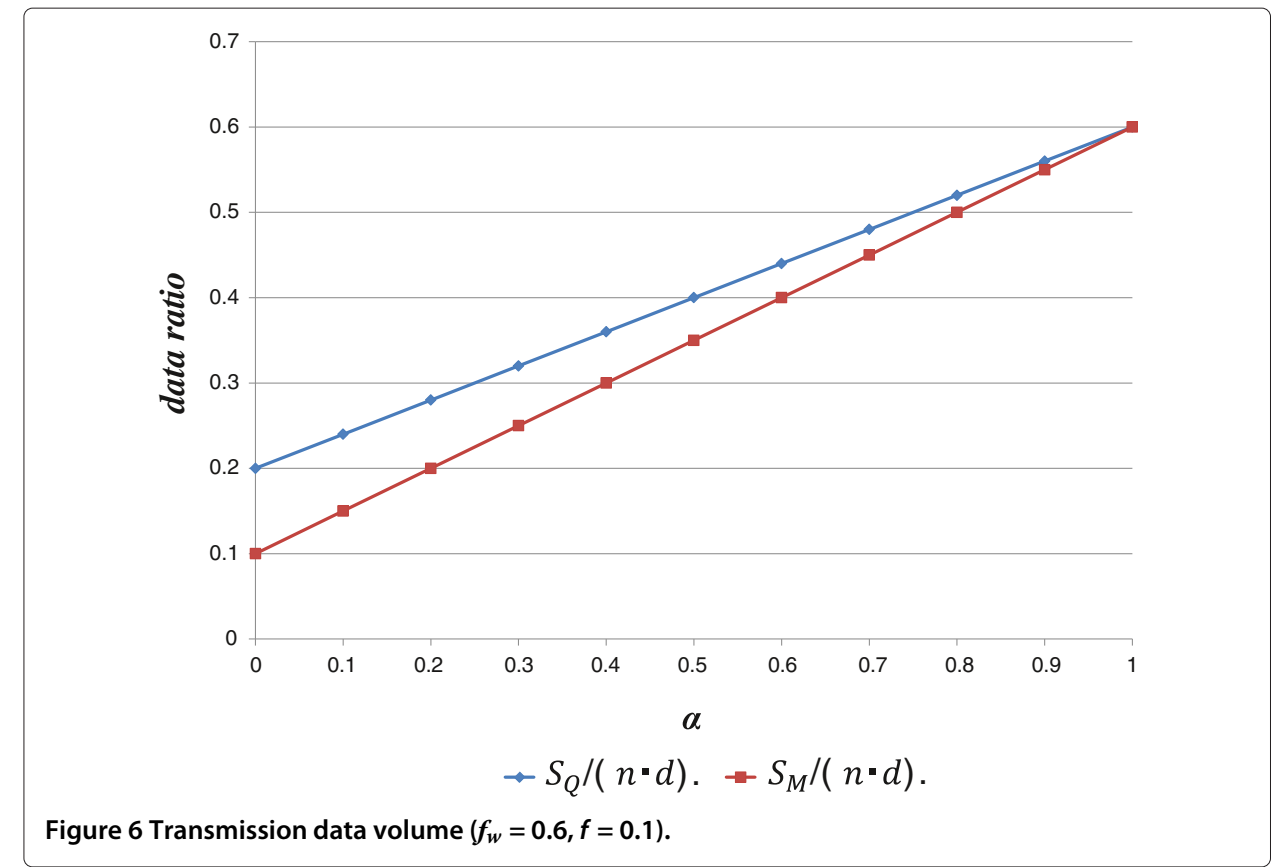

messages are transmitted for read and write in the MQB protocol, respectively, as well as the QB protocol. The volume of data transmitted to write the replicas is $n \cdot f_{w} \cdot d$ in the traditional $\mathrm{QB}$ protocol and the MQB protocol. Here, let $\alpha$ be the ratio of the number of write operations to the total number of operations issued by transactions $(0 \leq \alpha \leq 1)$. " $\alpha=0$ " means every request is read and " $\alpha=1$ " shows every request is write. In the QB protocol, the expected volume $S_{Q}$ of data transmitted in each transaction is $\alpha \cdot n \cdot f_{w} \cdot d+(1-\alpha) \cdot n \cdot\left(1-f_{w}\right) \cdot f_{r} \cdot d$. The expected volume $S_{M}$ of data transmitted in

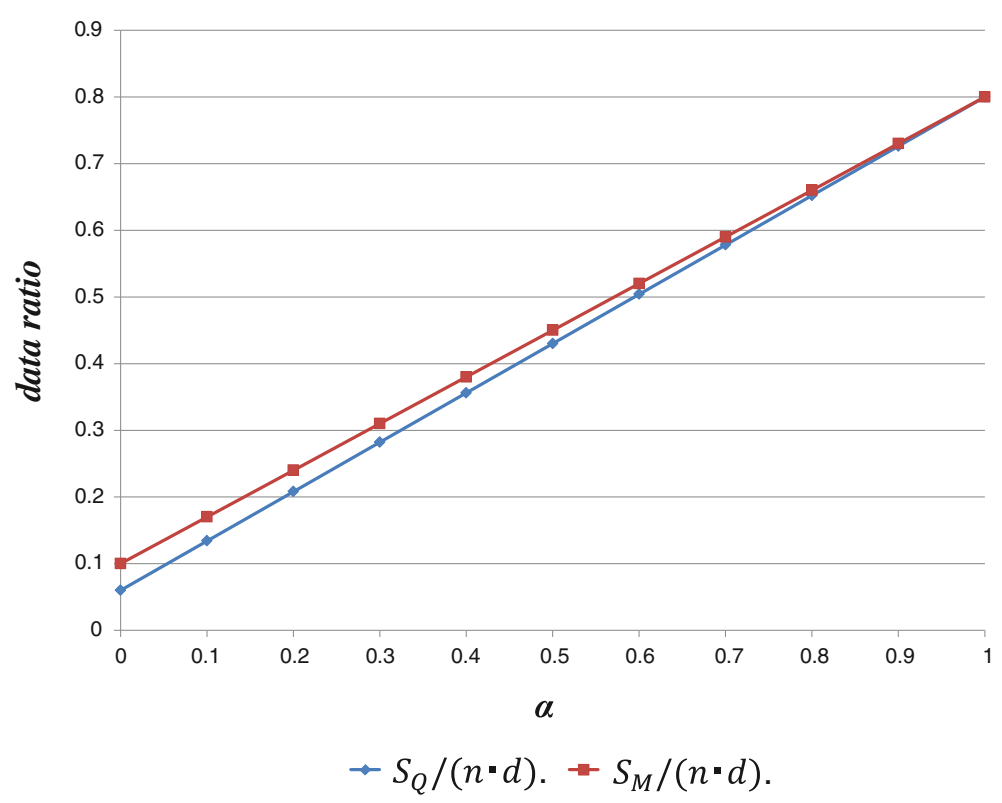

Figure 7 Transmission data volume $\left(f_{w}=0.8, f=0.1\right)$. 


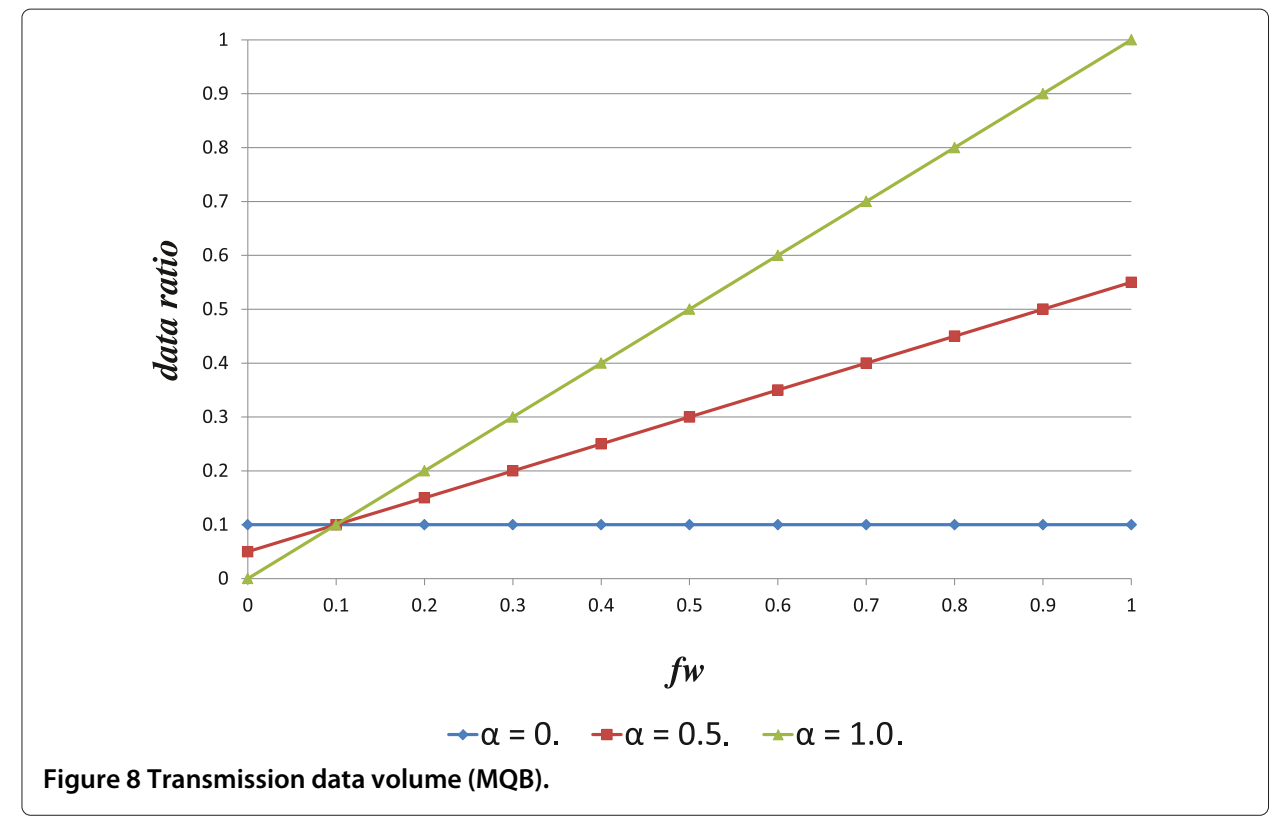

the MQB protocol is $\alpha \cdot n \cdot f_{w} \cdot d+(1-\alpha) \cdot d$ since no data is transmitted to every other replica in read than the top replica in the quorum $Q_{r}$.

Figures 6 and 7 show the ratios $S_{Q} /(n \cdot d)$ and $S_{M} /(n \cdot d)$ for the write ratio $\alpha$. Here, we assume there are ten replicas, $n=10$. In Figure $6, f_{w}=0.6$, and $f=0.1$. In Figure $7, f_{w}=$ 0.8 and $f=0.1 . S_{Q}=S_{M}$ for $\alpha=1$. As shown in Figures 6 and 7, the total amount of data transmitted can be reduced in the MQB protocol compared with the $\mathrm{QB}$ protocol. In Figure 8 , the ratio $S_{M} /(d \cdot n)$ is shown for the write probability $f_{w}$. Here, $f_{w}$ should be lager than 0.5 from the quorum constraint $\left(f_{w}>0.5\right)$. The lager $f_{w}$ and $\alpha$ are, the lager amount of data is transmitted. In order to reduce the communication overhead, the write quorum

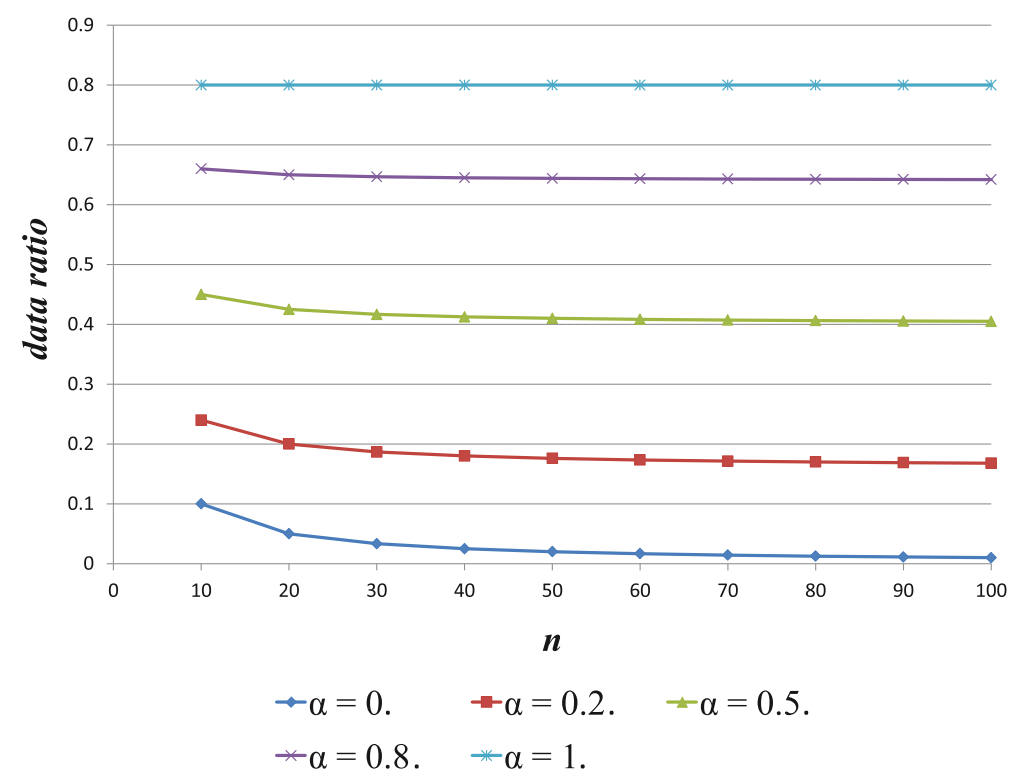

Figure 9 Transmission data volume (MQB) $\left(f_{w}=0.8, f=0.1\right)$. 


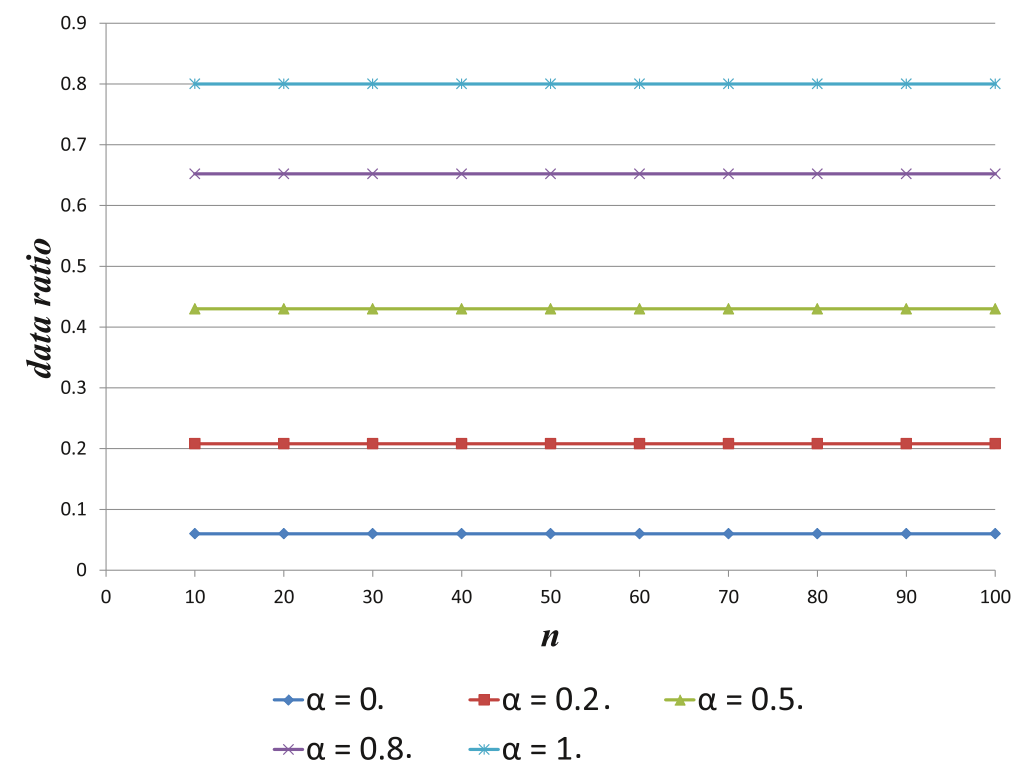

Figure 10 Transmission data volume (QB) $\left(f_{w}=0.8, f=0.1\right)$.

should be smallest. In Figure 9, the data ratio $S_{M} /(d \cdot n)$ for the number $n$ of replicas is shown where $f_{w}=0.8$. Figure 10 shows the data ratio $S_{Q} /(d \cdot n)$ for the number $n$ of the replicas. The communication overhead of the MQB protocol is increased in complexity $O(n)$ since the ratio to the number $n$ of the replicas is almost $O(1)$.

\section{Conclusions}

In this paper, we discussed the multimedia quorum-based (MQB) protocol to keep replicas of a multimedia object mutually consistent.A multimedia object is characterized in content and QoS parameters. Replicas are partially ordered in the newness-precedent relation $\preceq$ in terms of not only content parameter but also QoS parameters. If a replica $o_{i}$ has a larger vector value than another replica $o_{j}$, the replica i.e. $o_{i} . V>o_{j} . V, o_{i}$ is newer than $o_{j}$. A replica $o_{i}$ and the vector $o_{i} . V$ are updated each time the replica $o_{i}$ is manipulated. In order to increase the performance to read replicas, only the counter vector of each replica is updated in a quorum while the content and QoS parameters of the replica are not updated. We evaluated the $\mathrm{MQB}$ protocol in terms of the total volume of data transmitted among the replicas. We showed the total amount of data transmitted can be reduced in the MQB protocol compared with the traditional quorum-based (QB) protocol.

\section{Competing interests}

The authors declare that they have no competing interests.

\section{Author's contribution}

Tadateru Ohkawara carried out the MQB protocol studies, participated in designing, implementing, and evaluating the MQB protocol and drafted the manuscript. Ailixer Aikebaier and Tomoya Enokido participated in the design of the algorithm used in the MQB protocol. Makoto Takizawa conceived of the study, and participated in its design and coordination and helped to draft the manuscript. All authors read and approved the final manuscript.

\section{Acknowledgements}

This research is partially supported by the strategy research of Seikei University and MEXT, Grant-in-Aid for Building Strategy Research Infrastructure. 


\section{Author details}

1 Department of Computer and Information Science, Seikei University, 3-3-1 Kichijoji-kitamachi, Musashino-shi, Tokyo 180-8633, Japan. ${ }^{2}$ Faculty Of Business Administration, Rissho University, 4-2-16 Oosaki, Shinagawa-ku, Tokyo 141-8602, Japan. ${ }^{3}$ New Generation Network Laboratory, NICT, 4-2-1 Nukui-Kitamachi, Koganei, Tokyo 184-8795, Japan.

\section{Received: 17 October 2011 Accepted: 7 March 2012 Published: 10 May 2012}

\section{References}

1. Hofmann P, Woods D (2010) Cloud Computing: The Limits of Public Clouds for Business Applications. Journal of IEEE Internet Computing 14: 90-93 ISBN 1089-7801

2. Schollmeier R (2001) A Definition of Peer-to-Peer Networking for the Classification of Peer-to-Peer Architectures and Applications. In Proc. of the First International Conference on Peer-to-Peer Computing. Linkoping, Sweden, p 101

3. Gray J (1978) Notes on Database Operating Systems. Lecture Notes in Computer Science, vol 60. Springer Verlag

4. Helal A, Bhargava B (1995) Performance Evaluation of the Quorum Consensus Replication Method. In Proc. of the Internation Computer Performance and Dependability Symposium (IPDS'95). Erlangen, Germany, pp 165-172

5. Stoica I, Morris R, Karger D, Frans Kaashoek M, Balakrishnan H (2001) Chord: A Scalable Peer-to-peer Lookup Service for Internet Applications. In Proc. of ACM the 2001 Conference on Applications, Technologies, Architectures, and Protocols for Computer Communications (SIGCOMM '01). San Diego, califonia, USA, pp 149-160

6. Lakshman A, Malik P (2010) Cassandra: A Decentralized Structured Storage System. ACM SIGOPS Operating Systems Review 44(26): 35-40

7. Ohkawara T, Aikebaier A, Enokido T, Takizawa M (2011) Quorums-based Replication of Multimedia Objects in Distributed Systems. In Proc. of the International Conference on Network-Based Information Systems, NBiS2011, CD-ROM. Tirana, Albania

8. Enokido T, Higaki H, Takizawa M (1998) Group Protocol for Distributed Replicated Objects. In Proc. of the 27th International Conference on Parallel Processing (ICPP-98). Minneapolis, Minnesota, USA, pp 570-577

9. Tanaka K, Takizawa M (2001) Quorum-Based Locking Protocol for Replicas in Object-Based Systems. In Proc. of IEEE the 5th International Symposium or Autonomous Decentralized Systems. Dallas, Texas, USA, pp 196-203

doi:10.1186/2192-1962-2-11

Cite this article as: Ohkawara et al:: Quorums-based Replication of Multimedia Objects in Distributed Systems. Humancentric Computing and Information Sciences 2012 2:11.

\section{Submit your manuscript to a SpringerOpen ${ }^{\circ}$ journal and benefit from:}

$\checkmark$ Convenient online submission

- Rigorous peer review

- Immediate publication on acceptance

- Open access: articles freely available online

- High visibility within the field

- Retaining the copyright to your article

Submit your next manuscript at $\boldsymbol{\triangleright}$ springeropen.com 